\title{
Psychological Aspect of Pre-competition Warm-up: Motivational Process and Outcomes
}

\author{
Eylem Gencer (Corresponding author) \\ Department of Coaching Education, School of Physical Education and Sports \\ Kırşehir Ahi Evran University, 40100, Kırşehir, Turkey
}

Tel: 90-386-280-4773Ｅ mail: egencer@ahievran.edu.tr

Received: April 15, 2021 Accepted: May 17, 2021 Published: May 31, 2021

doi:10.5296/jei.v7i1.18535 URL: https://doi.org/10.5296/jei.v7i1.18535

\begin{abstract}
Pre-competition warm-up has mostly been examined physically in the literature, focusing on its effect on performance and protection from injuries. However, there is a lack of studies regarding the psychological influences of warm-up. Therefore, in this study it was aimed to explore the psychological reflections of pre-competition warm-up in terms of motivational process and outcomes. The research was designed in qualitative research, and phenomenology was employed. Participants were 10 international wrestlers whose age differentiated between 20 and 25 years. They have a sports career ranging from 8 to 12 years. The data were collected via focus group interviews and analyzed via content analysis. Results showed that wrestlers need pre-competition warm-up as they perceive it improves their performance and protects them from injuries. However, perception of the opponent as stronger or weaker, guides whether the warm-up is needed or not. In addition, pre-competition warm-up motivates wrestlers through stimulating, goal-directing (e.g., adaptation, concentration, awareness), and encouraging them to the competition. In conclusion, pre-competition warm-up not only physically prepares athletes for the competition, but also prepares them psychologically with its motivating influences.
\end{abstract}

Keywords: Psychology, Sports, Pre-competition warm-up, Motivation, Qualitative research

\section{Introduction}

The warm-up in sport is defined as a period of preparatory exercise to enhance subsequent competition or training performance (Hedrick, 1992). In another definition, the warm-up is described as a pre-exercise routine that includes improving muscle dynamics to reduce the risk of injury and preparing the athlete for the demands of exercise (Woods, Bishop, \& Jones, 2007; Taylor, Sheppard, Lee, \& Plummer, 2009). Based on the definitions, the main purpose 
of warming up is to improve training and competition performance and to minimize the risk of injury (Shellock \& Prentice, 1985). Pre-exercise warm up increases muscle and tendon suppleness, stimulates blood flow to the periphery, increases body temperature, and enhances free, coordinated movement (Smith, 1994). When the literature is examined, it is possible to collect the studies conducted on warm-up in two main groups. While the first group studies focus on the warm-up and performance (Zois, Bishop, Ball, \& Aughey, 2011; Burkett et al., 2005; Burnley et al., 2005; Faigenbaum et al., 2005, 2006; Fradkin et al., 2004; Koch et al., 2003; McMillian et al., 2006; Stewart \& Sleivert, 1998; Thompsen et al., 2007; Fletcher \& Jones, 2004; Neiva et al., 2015; Needham et al., 2009), the second group studies focus on the warm-up and sports injuries (Herman et al., 2012; Labella et al., 2011; Fradkin et al., 2007; Woods et al., 2007; Grooms et al., 2013; McCrary et al., 2015).

Although the studies regarding warm-up are physically based, the psychological aspect of the warm-up cannot be ignored. In other words, pre-competition warm-up not only provides physical preparation, but also can contribute to psychological preparation. As some researchers suggested, warm-up may include a psychological influence (Bishop, 2003), and a well-designed warm-up can assist the athlete in mentally focusing on the upcoming task and to bring about various physiological changes to optimize performance (Swanson, 2006). In addition, there are some other suggestions that warm-up may provide valuable time for athletes to mentally prepare for their event and may improve performance by providing time to concentrate (Shellock \& Prentice, 1985; Gourgoulis et al., 2003; McGowan, Pyne, Thompson, \& Rattray, 2015). Gencer and Tunçel (2018) put forward that warm-up has a role in psychological preparation of athletes. However, although there are some hypotheses stating that warming up may have psychological reflections, there is a lack of empirical research examining the psychological outcomes of warm-up in the context of exercise and sport psychology.

Understanding and enhancing motivation is one of the main research topics in exercise and sport psychology. Motivation concerns energy, direction, and persistence (Ryan \& Deci, 2000) and tries to explain how and why goals emerge and how these goals are maintained (Frey \& Jegen, 2001). Motivation is not an entity, but is a process (Maehr \& Braskamp, 1986). Hence, to understand the motivation, the constructs that drive the process need to be understood (Roberts, Treasure, \& Conroy, 2007). Motivational process is defined by the psychological constructs that energize, direct, and regulate achievement behavior (Roberts, Treasure, \& Conroy, 2007). These constructs are personal or socially valued goals, emotional arousal, and personal agency beliefs (Ford, 1992). The individual develops personal goals within any achievement context, and it is these personal goals that give meaning to achievement striving and energize subsequent action. Personal goals reside in the person, but they may be derived from context cues (Ames, 1992) or through instructions given by significant others (Locke \& Latham, 1990). The personal goals may also be culturally or socially defined (Maehr \& Nicholls, 1980) but will be useful as a part of a motivational process only if the person adopts them. Clearly, it is these personal and/or socially valued goals that often energize achievement striving (Roberts, 2001). Personal agency beliefs direct and regulate the ongoing stream of achievement behavior. Personal agency beliefs may be based on one's beliefs in 
one's capability to attain the goal or on evaluation of the context to support one's goal striving (Roberts, 2001). Emotional arousal may serve as a source of energy or evaluation to affect achievement striving (Ford, 1992).

Motivation theories focus on what energizes and gives direction to behavior (Ryan \& Deci, 2017). In the literature, achievement behavior, which motivation theories purport to explain, has typically been defined as behavioral intensity (trying hard), persistence (continuing to try hard), choice of action possibilities, and performance (outcomes). In physical activity settings, achievement behaviors are those behaviors witnessed when participants try harder, concentrate more, persist longer, pay greater attention, perform better, choose to practice longer, and/or join or drop out of physical activities (Roberts, 2001). The determinants of achievement behavior are approach and/or avoidance dispositions, expectancies, incentive values of success and failure, and/or cognitive assessments of success and failure, including the possibility of succeeding or failing (Roberts, 2001). The concept of motivation has been discussed in terms of deterministic to mechanistic and organismic to cognitive theories in the historical process (Ford, 1992). Deterministic and mechanistic theories assume humans as passive and driven by psychological needs or drives. Organismic theories acknowledge innate needs but also recognize that a dialectic occurs between the organism and the social context. Cognitive theories view humans as active and initiating action through subjective interpretation of the achievement context (Roberts, Treasure, \& Conroy, 2007). Contemporary theories assume that the human is an active participant in decision making and in planning achievement behavior (e.g., Bandura, 1986; Deci \& Ryan, 1985; Dweck \& Leggett, 1988; Kuhl, 1986; Maehr \& Nicholls, 1980; Nicholls, 1989).

The majority of motivation research in sport have adopted a social-cognitive approach (e.g., Duda, 1992, 2001; Duda \& Hall, 2001; Duda \& Whitehead, 1998; Roberts, 1984, 1992, 2001; Roberts, Treasure, \& Kavussanu, 1997) where the achievement is defined as the attainment of a personally or socially valued achievement goal that has meaning for the person in a physical activity context (Roberts, Treasure, \& Conroy, 2007). Achievement is subjectively defined, and success or failure in obtaining the goal is a subjective state based on the participant's assessment of the outcome of the achievement behavior (Maehr \& Nicholls, 1980; Spink \& Roberts, 1980).

Achievement goal theory (Duda, 2005; Duda \& Hall, 2001; Roberts, 2001; Roberts et al., 1997) assumes that the individual is an intentional, goal-directed organism who operates in a rational manner, and that achievement goals govern achievement beliefs and guide subsequent decision making and behavior in achievement contexts. It is argued that to understand the motivation of individuals, the function and meaning of the achievement behavior to the individual must be considered and the goal of action understood. Individuals give meaning to their achievement behavior through the goals they adopt. It is these goals that reflect the purposes of achievement striving. Once adopted, the achievement goal determines the integrated pattern of beliefs that undergird approach and avoidance strategies, the differing engagement levels, and the differing responses to achievement outcomes (Roberts, Treasure, \& Conroy, 2007). 
In this respect, to understand the motivational process of pre-competition warm-up in wrestling, we need to understand how wrestlers perceive the pre-competition warm-up, and why they warm up. Accordingly, in this study, it was aimed to explore the motivational process and outcomes of pre-competition warm-up through international wrestlers' perceptions and experiences. With this study, the following questions were sought to answer generally:

(1) Do wrestlers need pre-competition warm-up? Why?

(2) Does pre-competition warm-up motivate wrestlers? How?

\section{Method}

\subsection{Research Design}

The study was designed in qualitative research and phenomenology was employed as it was tried to develop a deeper understanding about the motivational features of pre-competition warm-up (Creswell, 2007). The phenomenon of interest is "pre-competition warm-up". Phenomenology focuses on the phenomena that we are aware of but do not have understanding broadly and deeply. Phenomena can emerge in various forms such as events, experiences, perceptions, orientations, concepts, and situations (Yıldırım \& Şimşek, 2005). The basic purpose of phenomenology is to reduce individual experiences with a phenomenon to a description of the universal essence (Creswell, 2007). This description consists of "what" individuals experienced and "how" they experienced it (Moustakas, 1994).

\subsection{Participants}

The sample of the study was composed two focus groups of $5(\mathrm{n}=10)$ international wrestlers determined by criterion sampling, which is one of the purposive sampling (Miles \& Huberman, 1994). The criteria as being "international" was determined by the researcher in order to find out the perceptions of elite wrestlers especially. Even there are different suggestions on the number of eligible participants for focus group interviews (Goss \& Leinbach, 1996; Morgan, 1997; Gibbs, 1997; Edmunds, 2000), Kitzinger (1995) suggest that the eligible number of participants for focus group interviews vary between 4 to 9 . The ages of the participants ranged from 20 to 25 years. Participants are active in sports. The career average of the participants was 10.4 years. Participants also attend higher education institutions. The identities of the participants were hidden in accordance with the principles of the research design and to make them answer the research questions sincerely. Instead, all wrestlers were given pseudonyms as "featherweight", "lightweight", "welterweight", "middleweight", and "heavyweight".

\subsection{Data Collection Tool}

The data was obtained via focus group interview (Stewart \& Shamdasani, 1990; Kitzinger, 1994, 1995; Krueger, 1994; Gibbs, 1997; Bowling, 2002). The reason for preferring focus group interview was to create an interactive environment and thus obtain richer data (Kitzinger, 1994, 1995; Patton, 2002). A semi-structured interview approach was employed. In the process of preparing the questions included in the interview form, two ways were 
followed: First; literature was investigated. Variables that were thought to be related to motivation were determined and these variables were formed into semi-structured, open-ended questions via a pilot study with 5 elite wrestlers. Latter: 1 lecturer from a Psychological Counseling Guidance Department and 1 lecturer from a Physical Education and Sports Department who have qualitative research examined the interview form. They added and excluded some questions. Thus, the final shape of the interview form was given. Sample questions in the interview form were as follows:

Do you need the warm-up before the competition? Why?

Does the pre-competition influence your motivation? How?

How did you feel when you warmed up and not warmed up or insufficiently warmed up before the competition? Were there any differences?

Does the opponent influence your decision of warm up or not?

\subsection{Procedure}

Permission was obtained from the Research Ethics Committee of Kurşehir Ahi Evran University for the study. After receiving the ethics committee approval for the research, the international wrestlers were contacted, the purpose of the investigation was explained and the permission for the interviews were obtained. Elite wrestlers who had been granted permission for the interviews, were invited to the office of the researcher at the predetermined date and time. Participants were informed about the purpose and the content of the investigation prior to the interviews. All participants provided informed consent. Interviews were conducted in the office of the researcher and moderated by him. A semi-structured interview approach was employed, and the moderator adopted a conversational approach to allow new themes to emerge (Potter \& Hepburn, 2005) and the participants were asked to talk as widely as possible regarding the process of pre-competition warm-up they had experienced before. Interviews with the participants were recorded with a tape recorder by permission from elite wrestlers. The interviews could not be recorded with a video recorder due to the abstention of some participants. In addition, notes were made by the researcher during the interviews. The interviews lasted 80 minutes with the first group and 87 minutes with the second group.

\subsection{Data Analysis}

The data obtained in the research was analysed via content analysis. In phenomenological studies, data analysis is carried out to reveal experiences and meanings (Yıldırım \& Şimşek, 2005). In this respect, the interviews transcribed verbatim. The interview transcripts were read many times and significant statements of wrestlers were highlighted to provide an understanding of how the participants experienced the pre-competition warm-up (i.e., horizonalization) (Moustakas, 1994). Then, it was developed clusters of meaning from wrestlers' significant statements into themes. These significant statement and themes were then used to write a description of what the wrestlers experienced (i.e., textural description) (Creswell, 2007). They were also used to write a description of the context that influenced how the wrestlers experienced the pre-competition warm-up (i.e., structural description) 
(Creswell, 2007). From the structural and textural description, a composite description that presents the essence of the phenomenon was reported.

\subsection{Validity and Reliability}

Lincoln and Guba (1985) adopt the concept of trustworthiness rather than validity and reliability in qualitative research and suggest some strategies to enhance the quality of qualitative research (e.g., Credibility, transferability, dependability, confirmability). To ensure the credibility of the research, a prolonged engagement (Lincoln \& Guba, 1985) between the researcher and the community was established. The researcher is an old international wrestler, and he has a general understanding of the culture, social settings, and the phenomenon of interest by spending and experiencing adequate time. A developed relationship and rapport with the members of the culture had already existed. Besides, a pilot study was carried out with 5 international wrestlers. In addition, peer debrief (Creswell \& Miller, 2000) was conducted throughout the study by a lecturer who has qualitative research. Subsequently, participants were asked soon after the interviews whether the data obtained in the course of the interviews reflected their expressions and they were given the opportunity to add and exclude expressions; so that the accuracy of the obtained data was checked (e.g., member check) (Erlandson et al., 1993). To ensure the transferability of the research the purposive sampling (Erlandson et al., 1993) was adopted. So, the phenomenon of interest and the method of the research can be applied in other contexts. In addition, by a thick description (Creswell \& Miller, 2000; Lincoln \& Guba, 1985), the settings, participants and the themes of the study were described in detail. To establish dependability and confirmability, the researcher described the process of obtaining and analysis of data, interpreting and creating themes to an external auditor who has qualitative research and not involved in the research (i.e., inquiry audit, audit trail) (Lincoln \& Guba, 1985; Creswell \& Miller, 2000). They discussed and evaluated whether the findings, interpretations and the conclusions were supported by the data.

\section{Results}

In this section, the reasons for the wrestlers to warm up before the competition, the motivational outcomes of the pre-competition warm-up are included, and the themes obtained in the research are interpretated through the wrestlers' expressions.

\subsection{Reasons to Warm up}

\subsubsection{Performance Improvement}

The results revealed that the wrestlers warm up for various purposes. However, it is possible to collect the reasons to warm up in two main objectives. The first objective is performance improvement. Some athletes consider that pre-competition warm-up is associated with starting the competition prepared. With the warm-up, they can make a better start to the competition and thus they can be more effective. When they start the match without warming up and/or insufficiently warm-up, they may experience unexpected loss of points due to not warming up and/or insufficient warming up. A participant's experience regarding theme as follows: 
Of course, I perform better when I warm up. Because my body gets ready for the competition. For example, I would not warm up adequately when I was in the junior category and my opponents would get points from me during the first period of 1- or 1,5-minutes time. Then, I decided to warm up efficiently and by this way, my opponents could not get any points from me. Some of them, however, could get points at the first period of the competition. But it was not because of my not warming up; that was the common loss of points, happening through the competition. But it was me who gave points to opponents during the first period of the competition when I did not warm up sufficiently. (Welterweight)

Besides, starting the match without warming up, or with insufficient warm up also brings some negative consequences. Some may exhibit an ineffective performance due to insufficient or not warming up in the first period. Even if they feel warmed up in the second half of the competition, it may be too late and may lose the game in the first half. Another participant's experience is as follows:

When I start the match by warming up, I begin the first period of competition more prepared. I perform better when I warm up. When I do not warm up, I can perform well at the second half of the game, yet this time the opponent can win the match at the first half of the competition. (Heavyweight)

The second objective of warming up is the facilitating influence of warm-up in the performing of techniques. Some wrestlers perceive pre-competition warm-up as a requirement to demonstrate the desired performance during the competition. They think that the warm-up helps them get rid of the stiffness and thus they perform better during the competition. Otherwise, they can only feel this situation as the match is over. Because they feel warmed up only when the match is over. A participants' quotes as follows:

In my opinion, the warm-up is necessary. When I begin the match without warming up, my body can not apply techniques, I mean your body does not allow you to perform effectively even if you could do it when warmed up. I feel like I will be able to play three more games when the match is over. I feel as if there is an obstruction, and the warm-up removes it. I perform much better when I warm up. (Lightweight)

Pre-competition warm-up also provides a fluency in performing the techniques. Thus, the wrestlers can demonstrate the performance they want throughout the competition. While the warmed-up wrestlers experience this situation throughout the competition, the unwarmed wrestlers only experience this situation towards the end of the competition. A middleweight wrestler's perception is as follows:

In a 6-minutes match, if the wrestlers start the match by warming up, a hard match is played during these 6 minutes period and the techniques are performed perfectly. The warmed wrestler performs smoothly from the beginning to the end of the match, while the unwarmed wrestler performs indeterminedly on the other hand. (Middleweight)

Pre-competition warm-up also affects the number of attempts to use techniques during the match. While the warmed wrestlers have more attempts to use the techniques in the 
competition, the unwarmed wrestlers have less attempts. A featherweight wrestler's perception is as follows:

When I do not warm up, I cannot apply the technique which I can easily apply by warming up. In other words, I can attempt only once to get points instead of 10 times. But, if I warm up sufficiently, no matter how the opponent is talented, I can perform perfectly. (Featherweight)

As can be understood from the wrestlers' statements, pre-competition warm-up prepares athletes physically for the competition by improving muscle and joint dynamics and so they start the competition more prepared and perform techniques smoothly. On the other hand, not warming up declines the techniques to be performed and prevents them from starting the match as they desire.

\subsubsection{Prevention from Injuries}

Another reason for warming up before the competition was explored to be protection from injuries. Some wrestlers also need the pre-competition warm-up in order not to get injured in the competition. They think that they may suffer injury when they compete without warming up in the competition. This thought pushes them to compete more cautiously in the competition. A middleweight wrestler states the situation as follows:

The warming up is necessary for not being injured as well. If you start the match without warming up, an injury may occur suddenly. This means the wrestler is getting prone to being injured. When I start the match without warming up, I am worried that I may be injured. So, I wrestle hesitantly with the fear of being injured. (Middleweight)

At the basis of this perception lies the injuries they experienced during training or competition they had previously come out without warming up. These negative experiences in the past are an important reason that makes athletes conclude that pre-competition warm-up is a need and makes them warm up. A lightweight wrestler's statement is as follows:

An athlete who does not warm up before the competition is always worried about potential injuries occurring during the match. Because each athlete got absolutely injured in a training or competition session due to not warming up. Therefore, all athletes need the warm-up before the competition. (Lightweight)

Although wrestlers need pre-competition warm-up owing to its performance influences and prevention from injuries, perception of the opponent determines whether warm-up is needed or not. In other words, perception of opponent determines the approach and avoidance dispositions of the athletes to warm up or not. The approach to the strong opponent differs among wrestlers. While some of the wrestlers believe that sufficient warm-up is needed before the competition against the strong opponent to perform better, some do not feel the necessity to warm up, thinking that the strong opponent will easily defeat them. Some statements of wrestlers as follows:

Suppose that, I am going to play with an elite wrestler. The better opponent I play with, 
the more efficiently I should warm up. So, I can perform better. (Middleweight)

If you wrestle with an elite wrestler, the warming up is crucial. Elite opponent shows his reaction more effectively and faster. That is why you should warm up. (Featherweight)

But there is a case, if your opponent is better than you, you think there is no way to win the match if you are warmed up or not. You think he will beat you anyway. (Welterweight)

Similarly, wrestlers, who see their opponents weaker than themselves, do not consider pre-competition warm-up before the match as a need, thinking that they will beat their opponents in the competition anyway. Some expressions of wrestlers as follows:

If I am sure that I will win the match, I do not need to warm-up so much. Because I will already win the match. (Lightweight)

If I wrestle with a strong wrestler, I warm up efficiently. But, if he is not, I do not warm up as much. (Heavyweight)

\subsection{Motivational Outcomes}

\subsubsection{Stimulation}

Pre-competition warm-up affects the arousal levels of the wrestlers. Some factors arising from the competition environment such as waiting for the match can reduce the arousal levels of some wrestlers. The decrease in arousal also can causes athletes to feel lazy and sleepy. Pre-competition warm-up eliminates this undesirable situation, energizing the wrestlers and helping them recover. Thus, it helps the wrestlers to start the competition with a higher arousal level. Some statements of wrestlers as follows:

I need the warm-up to get rid of the deadness, I have to be awakened. Sometimes, I am getting sleepy while I am waiting for the match. That is because of the environment. (Welterweight)

When I warm up, I blow away the cobwebs on me. But, when I start the match without warming up, I lose the first half. That is due to not warming up. I have difficulties to be awake in this circumstance. (Middleweight)

Everybody gets vigorous after warming up and feels strong as a bull. The ambience of the competition affects athletes negatively. Thus, warming up is needed. (Featherweight)

\subsubsection{Goal-Direction}

Pre-competition warm-up also affects the cognitive processes of the wrestlers. To compete without warming up prevents some wrestlers from adapting to the competition and causes them to be interested in other things in the environment. Pre-competition warm-up helps the wrestlers to adapt to the competition and enables them to focus on their goals. A heavyweight wrestler's experience is as follows:

I took part in a competition in Çorum. I did not warm up before the match. At the first 
half, I was looking around to see who there were at the tribune. I was interested in other things. There was nothing related to the match in my mind. I failed to adapt to that environment. The point is that warming up makes you adapt to that environment. (Heavyweight)

Besides, when some wrestlers compete without warming up, they may ignore the importance of the environment and competition they are in. Pre-competition warm-up creates awareness of the importance of the competition and so it directs the wrestlers to the competition. A middleweight wrestler's statement is as follows:

I was in the Turkey Championship. I had behaved as if I had been in Province Selection when I did not warm up. But, when I do, I am aware of the importance of the match happening there. (Middleweight)

In addition, pre-competition warm-up helps wrestlers to concentrate on the competition. Some wrestlers stated that when they participate in the competition without warming up, they cannot concentrate on the competition and the lack of concentration negatively affects their performance. A lightweight wrestler's statement is as follows:

I started the match without warming up in the Turkey Championship. After 1.5 minutes of the start of the match, I was wrestling so badly and looking around like a rookie. I was warming up newly when the score was 4-0 in my opponent's favor. I mean when I do not warm up, I cannot concentrate on the match. (Lightweight)

\subsubsection{Encouragement}

Pre-competition warm-up increases the desire of the athletes for the competition. They emphasize that they are more eager for the match when they warm up, and they are more reluctant when they do not warm up. In other words, pre-competition warm-up motivates athletes for the competition through regulating their emotional mood. Some participants' expressions for the theme as follows:

I need to warm up. When I do it, I am getting so motivated and eager. This is the only way to be motivated for me. (Heavyweight)

If I do not warm up before the match, I do not have a desire to play. But when I warm up, I feel more willing. (Welterweight)

If I am not warmed up, I am not eager for the match. But, If I am, I am getting eager. (Lightweight)

\section{Discussion and Conclusion}

In this research, the motivational process and outcomes of pre-competition warm-up were tried to be explored. Results showed pre-competition warm-up was needed by the wrestlers for two reasons. The first of these is the perception that pre-competition warm-up improves performance. Some wrestlers need pre-competition warm-up owing to its performance improving influences. The second reason is the perception that pre-competition protects from injuries. However, the perception of the opponent as stronger or weaker determines whether 
pre-competition warm-up is needed or not. In addition, the pre-competition warm-up motivates wrestlers through stimulating, goal-directing, and encouraging them to the competition.

To explore the motivational process of pre-competition warm-up, we need to consider the function and meaning of the pre-competition warm-up to the wrestlers and need to understand the goal of warm-up (Roberts, 2001; Roberts, Treasure, \& Conroy, 2007). According to social cognitive approach, achievement is defined as the attainment of a personally or socially valued achievement goal that has meaning for the person in a physical activity context (e.g., defeating an opponent, improving a skill) (Roberts, Treasure, \& Conroy, 2007). Motivational process is discussed in this approach around the components of energization, direction, and regulation (Roberts, Treasure, \& Conroy, 2007). The psychological constructs that energize, direct, and regulate achievement behaviour are personal goals, personal agency beliefs, and emotional arousal (Ford, 1992; Roberts, 2001). Among these, personal goals give meaning and energize to achievement behavior of individuals (Roberts, 2001). From this point of view, pre-competition warm-up may be conceptualized as the energizing force. Because wrestlers give a meaning to the pre-competition warm-up as improving their performance and protecting them from injuries. The main goal in wrestling competition is defeating an opponent. Wrestlers believe that they can attain this goal through pre-competition warm-up, which helps them to demonstrate competence and protects them from injuries.

Although wrestlers need the pre-competition warm-up owing to its performance enhancing influences and prevention from injuries, perception of the opponent guides whether warm-up is needed or not. When athletes face a better opponent, while some of them need warming up to show better performance during the competition, the others do not see warming up as a need, thinking that the good opponent will beat them anyway. On the other hand, when faced with a weaker opponent, they do not need to be warmed up that much because they think they will beat their opponent anyway. These findings can be conceptualized within the framework of personal agency beliefs/perceived competence (Roberts, 2001). Their beliefs that they can beat their opponents or not, direct and regulate the ongoing achievement behaviour. In other words, cognitive assessments of success and failure determine the decision of warming up or not. The relative influence of situation and personal sources of motivation and their interaction affect the decision of warming up or not (Roberts, 2001).

Regarding the motivational outcomes of pre-competition warm-up, it influences athletes' emotional arousal, it makes this impact through stimulating them. Emotional arousal has been discussed whether it is determinant of motivation or a consequence of assessments of the results of achievement striving (Roberts, 2001). In the current study, it can be considered as a result of achievement striving. Because some participants stated that the competition environment can create a state of sleepiness, and this mood disappears after the warm-up and they feel more energetic. In this respect, the pre-competition warm-up can be assumed as a motivating factor as it energizes their achievement behavior and regulates their emotional arousal. 


\section{MInstitute Macho $_{\text {Int }}$}

The other motivational outcome of pre-competition warm-up is goal-direction. Pre-competition warm-up makes athletes adapt and focus to the competition and creates awareness of the importance of the competition. Although the psychological constructs drive the motivational process (Roberts, 2001), the competition ambiance may sometimes negatively affect this process. As some participants stated, waiting for the match may demotivate athletes. After they warm up, they feel more adapted, focused, and conscious. From this point of view, the pre-competition warm-up can prepare athletes psychologically to the competition, allowing them to concentrate, adapt, be conscious; in short, direct to competition.

The last motivating outcome of pre-competition warm-up is that it increases the desire of athletes to the competition. They feel more eager after the warming up. Warming up induce the approach dispositions of athletes to the competition (Roberts, 2001). In this respect, it can be assumed as a motivating factor regulating their emotions to the competition, and so it prepares them to the competition psychologically.

This research brings a new perspective to the motivational process. Motivational process is driven by the psychological constructs that energize, direct, and regulate achievement behavior (Roberts, 2001). These psychological constructs are accepted as personal goals, personal agency beliefs and emotional arousal (Roberts, 2001). Although it is thought that these structures initiate the motivational process, it should not be ignored that individuals may become demotivated in the process due to various environmental conditions. In such a situation, pre-competition warm-up can reactivate the process by stimulating, goal-directing, and encouraging athletes. It does not mean that athletes do not have goals, competencies, or arousal. This simply means athletes are sometimes demotivated due to various factors arising from the competitive environment.

The results of the research present some important implications to the researchers and the practitioners in the field. Pre-competition warm-up is examined and discussed physically based and assumed to prepare athletes physically regarding performance improvement and protection prom injuries. However, with this study it was explored that pre-competition warm-up is an energizing force as it is perceived to improve performance and protect from injuries. Besides, it also prepares athletes psychologically to the competition through motivating influences. It has stimulating, directing, and encouraging outcomes. In this respect, pre-competition warm-up not only prepares athletes physically, but also it prepares them psychologically to the competition through motivating them.

The study was conducted with elite wrestlers. So, the results of the research can be evaluated regarding wrestling. However, the pre-competition warm-up is performed almost in all sports. So, the results can give some clues about the potential outcomes of pre-competition warm-up. Examining the psychological features of pre-competition warm-up in different sports can contribute to a better understanding of the psychological preparation aspect of pre-competition warm-up. 


\section{References}

Ames, C. (1992). Achievement goals, motivational climate, and motivational processes. In G.c. Roberts (Ed.), Motivation in sport and exercise (pp. 161-176). Champaign, IL: Human Kinetics.

Bandura, A. (1986). Social foundations of thought and action: A social cognitive theory. Englewood Cliffs, NJ: Prentice-Hall.

Bishop, D. (2003). Warm-up II. Performance changes following active warm-up and how to structure the warm-up. Sports Medicine, 33(7), 483-498. https://doi.org/10.2165/00007 256-200333070-00002

Bowling, A. (2002). Research methods in health: Investigating health and health services. Philadelphia, PA: McGraw-Hill House.

Burkett, L. N., Phillips, W. T., \& Ziuraitis, J. (2005). The best warm-up for the vertical jump in college-age athletic men. The Journal of Strength \& Conditioning Research, 19(3), 673-676. https://doi.org/10.1519/00124278-200508000-00031

Burnley, M., Doust, J. H., \& Jones, A. M. (2005). Effects of prior warm-up regime on severe-intensity cycling performance. Medicine and Science in Sports and Exercise, 37(5), 838-845. https://doi.org/10.1249/01.MSS.0000162617.18250.77

Creswell, J. W. (2007). Qualitative inquiry and research design: Choosing among five approaches. Lincoln: Sage Publications.

Creswell, J. W., \& Miller, D. L. (2000). Determining validity in qualitative inquiry. Theory into Practice, 39(3), 124-130. https://doi.org/10.1207/s15430421tip3903_2

Deci, E. L., \& Ryan, R. M. (1985). Intrinsic motivation and self-determination in human behavior. New York: Plenum Press. https://doi.org/10.1007/978-1-4899-2271-7

Duda, J. L. (1992). Motivation in sport settings: A goal perspective approach. In G. C. Roberts (Ed.), Motivation in sport and exercise (pp. 57-91). Champaign, IL: Human Kinetics.

Duda, J. L. (2001). Achievement goal research in sport: Pushing the boundaries and clarifying some misunderstandings. In G. C. Roberts (Ed.), Advances in motivation in sport and exercise (pp. 129-182). Champaign, IL: Human Kinetics.

Duda, J. L. (2005). Motivation in sport: The relevance of competence and achievement goals. In A. J. Elliot \& C. S. Dweck (Eds.), Handbook of competence and motivation (pp. 318-335). New York: Guilford Press.

Duda, J. L., \& Hall, H. (2001). Achievement goal theory in sport: Recent extensions and future directions. In R. N. Singer, H. A. Hausenblas, \& C. M. Janelle (Eds.), Handbook of sport psychology (2nd ed., pp. 417-443). New York: Wiley.

Duda, J. L., \& Whitehead, J. (1998). Measurement of goal perspectives in the physical domain. In J. L. Duda (Ed.), Advances in sport and exercise psychology measurement (pp. 
21-48). Morgantown, WV: Fitness Information Technology.

Dweck, C. S., \& Leggett, E. L. (1988). A social-cognitive approach to motivation and personality. Psychological Review, 95(2), 256-273. https://doi.org/10.1037/0033-295X. 95.2.256

Edmunds, H. (2000). The focus group research handbook. New York: McGraw-Hill.

Erlandson, D. A., Harris, E. L., Skipper, B. L., \& Allen, S. D. (1993). Doing naturalistic inquiry: A guide to methods. Sage Publications.

Faigenbaum, A. D., Bellucci, M., Bernieri, A., Bakker, B., \& Hoorens, K. (2005). Acute effects of different warm-up protocols on fitness performance in children. The Journal of Strength \& Conditioning Research, 19(2), 376-381. https://doi.org/10.1519/00124278-200505 000-00023

Faigenbaum, A. D., McFarland, J. E., Schwerdtman, J. A., Ratamess, N. A., Kang, J., \& Hoffman, J. R. (2006). Dynamic warm-up protocols, with and without a weighted vest, and fitness performance in high school female athletes. Journal of Athletic Training, 41(4), 357-363.

Fletcher, I. M., \& Jones, B. (2004). The effect of different warm-up stretch protocols on 20 meter sprint performance in trained rugby union players. The Journal of Strength \& Conditioning Research, 18(4), 885-888. https://doi.org/10.1519/00124278-200411000-00035

Ford, M. E. (1992). Motivating humans: Goals, emotions, and personal agency beliefs. Sage Publications. https://doi.org/10.4135/9781483325361

Fradkin, A. J., Cameron, P. A., \& Gabbe, B. J. (2007). Is there an association between self-reported warm-up behaviour and golf related injury in female golfers? Journal of Science and Medicine in Sport, 10(1), 66-71. https://doi.org/10.1016/j.jsams.2006.04.001

Fradkin, A. J., Sherman, C. A., \& Finch, C. F. (2004). Improving golf performance with a warm up conditioning programme. British Journal of Sports Medicine, 38(6), 762-765. https://doi.org/10.1136/bjsm.2003.009399

Frey, B. S., \& Jegen, R. (2001). Motivation crowding theory. Journal of Economic Surveys, 15(5), 589-611. https://doi.org/10.1111/1467-6419.00150

Gencer, E., Tunçel, M. (2018). The effect of pre-game warm-up on motivation and performance: A focus group interview on national wrestlers. $16^{\text {th }}$ International Sport Sciences Congress, Antalya.

Gibbs, A. (1997). Focus groups. Social Research Update, 19(8), 1-8.

Goss, J. D., \& Leinbach, T. R. (1996). Focus groups as alternative research practice: experience with transmigrants in Indonesia. Area, 28(2), 115-123.

Gourgoulis, V., Aggeloussis, N., Kasimatis, P., Mavromatis, G., \& Garas, A. (2003). Effect of a submaximal half-squats warm-up program on vertical jumping ability. The Journal of 
Strength \& Conditioning Research, 17(2), 342-344. https://doi.org/10.1519/00124278-200305 000-00021

Grooms, D. R., Palmer, T., Onate, J. A., Myer, G. D., \& Grindstaff, T. (2013). Soccer-specific warm-up and lower extremity injury rates in collegiate male soccer players. Journal of Athletic Training, 48(6), 782-789. https://doi.org/10.4085/1062-6050-48.4.08

Hedrick, A. (1992). Exercise physiology: Physiological responses to warm-up. Strength \& Conditioning Journal, 14(5), 25-27. https://doi.org/10.1519/0744-0049(1992)014\%3C0025: PRTWU\%3E2.3.CO;2

Herman, K., Barton, C., Malliaras, P., \& Morrissey, D. (2012). The effectiveness of neuromuscular warm-up strategies, that require no additional equipment, for preventing lower limb injuries during sports participation: A systematic review. BMC Medicine, 10(1), 75. https://doi.org/10.1186/1741-7015-10-75

Kitzinger, J. (1994). The methodology of focus groups: The importance of interaction between research participants. Sociology of Health \& Illness, 16(1), 103-121. https://doi.org/ 10.1111/1467-9566.ep11347023

Kitzinger, J. (1995). Qualitative research: Introducing focus groups, British Medical Journal, 311, 299-302. https://doi.org/10.1136/bmj.311.7000.299

Koch, A. J., O’bryant, H. S., Stone, M. E., Sanborn, K., Proulx, C., Hruby, J., ... Stone, M. H. (2003). Effect of warm-up on the standing broad jump in trained and untrained men and women. The Journal of Strength \& Conditioning Research, 17(4), 710-714. https://doi.org/ 10.1519/00124278-200311000-00014

Krueger, R. A. (1994). Focus groups: A practical guide for applied research. London: Sage.

Kuhl, J. (1986). Motivation and information processing: A new look at decision making, dynamic change, and action control. In R. M. Sorrentino \& E. T. Higgins (Eds.), Handbook of motivation and cognition: Foundations of social behavior (pp. 404-434). Guilford Press.

LaBella, C. R., Huxford, M. R., Grissom, J., Kim, K. Y., Peng, J., \& Christoffel, K. K. (2011). Effect of neuromuscular warm-up on injuries in female soccer and basketball athletes in urban public high schools: Cluster randomized controlled trial. Archives of Pediatrics \& Adolescent Medicine, 165(11), 1033-1040. https://doi.org/10.1001/archpediatrics.2011.168

Lincoln, Y. S., \& Guba, E. G. (1985). Naturalistic inquiry. Sage Publications. https://doi.org/ 10.1016/0147-1767(85)90062-8

Locke, E. A., \& Latham, G. P. (1990). A theory of goal setting and task performance. Englewood Cliffs, NJ: Prentice Hall.

Maehr, M. L., \& Braskamp, L. A. (1986). The motivation factor: A theory of personal investment. Lexington Books/DC Heath and Com.

Maehr, M. L., \& Nicholls, J. G. (1980). Culture and achievement motivation: A second look. In N. Warren (Ed.), Studies in cross-cultural psychology (Vol. 2, pp. 221-267). New York: 
Academic Press.

McCrary, J. M., Ackermann, B. J., \& Halaki, M. (2015). A systematic review of the effects of upper body warm-up on performance and injury. British Journal of Sports Medicine, 49(14), 935-942. https://doi.org/10.1136/bjsports-2014-094228

McGowan, C. J., Pyne, D. B., Thompson, K. G., \& Rattray, B. (2015). Warm-up strategies for sport and exercise: mechanisms and applications. Sports Medicine, 45(11), 1523-1546. https://doi.org/10.1007/s40279-015-0376-x

McMillian, D. J., Moore, J. H., Hatler, B. S., \& Taylor, D. C. (2006). Dynamic vs. static-stretching warm up: The effect on power and agility performance. The Journal of Strength \& Conditioning Research, 20(3), 492-499. https://doi.org/10.1519/00124278-200608 000-00006

Miles, M. B., \& Huberman, A. M. (1994). Qualitative data analysis: An expanded sourcebook (2nd ed.). Sage Publications, Inc.

Morgan, D. L. (1997). Focus groups as qualitative research. California: Sage Publications. https://doi.org/10.4135/9781412984287

Moustakas, C. (1994). Phenomenological research methods. Thousand Oaks, CA: Sage Publications. https://doi.org/10.4135/9781412995658

Needham, R. A., Morse, C. I., \& Degens, H. (2009). The acute effect of different warm-up protocols on anaerobic performance in elite youth soccer players. The Journal of Strength \& Conditioning Research, 23(9), 2614-2620. https://doi.org/10.1519/JSC.0b013e3181b1f3ef

Neiva, H. P., Marques, M. C., Barbosa, T. M., Izquierdo, M., Viana, J. L., Teixeira, A. M., \& Marinho, D. A. (2015). The effects of different warm-up volumes on the 100-m swimming performance: A randomized crossover study. The Journal of Strength \& Conditioning Research, 29(11), 3026-3036. https://doi.org/10.1519/JSC.0000000000001141

Nicholls, J. G. (1989). The competitive ethos and democratic education. Cambridge, MA: Harvard University Press.

Patton, M. Q. (1990). Qualitative evaluation and research methods (2nd ed.). Sage Publications, Inc.

Patton, M. Q. (2002). Two decades of developments in qualitative inquiry: A personal, experiential perspective. Qualitative Social Work, 1(3), 261-283. https://doi.org/10.1177/ 1473325002001003636

Potter, J., \& Hepburn, A. (2005). Qualitative interviews in psychology: Problems and possibilities. Qualitative Research in Psychology, 2(4), 281-307. https://doi.org/10.1191/ 1478088705 qp045oa

Roberts, G. C. (1984). Achievement motivation in children's sport. In J. Nicholls (Ed.), The development of achievement motivation (pp. 251-281). Greenwich, CT: JAI Press. 
Roberts, G. C. (1992). Motivation in sport and exercise: Conceptual constraints and convergence. In G. C. Roberts (Ed.), Motivation in sport and exercise (pp. 3-29).Champaign, IL: Human Kinetics.

Roberts, G. C. (2001). Understanding the dynamics of motivation in physical activity: The influence of achievement goals on motivational processes. In G. C. Roberts (Ed.), Advances in motivation in exercise and sport (pp. 1-50). Champaign, IL: Human Kinetics. https://doi.org/10.1002/9781118270011.ch1

Roberts, G. C., Treasure, D. C., \& Conroy, D. E. (2007). Understanding the dynamics of motivation in sport and physical activity: An achievement goal interpretation. In G. Tenenbaum \& R. C. Eklund (Eds.), Handbook of sport psychology (pp. 3-30). John Wiley \& Sons, Inc. https://doi.org/10.1002/9781118270011.ch1

Roberts, G. C., Treasure, D. C., \& Kavussanu, M. (1997). Motivation in physical activity contexts: An achievement goal perspective. In P. Pintrich \& M. Maehr (Eds.), Advances in motivation and achievement (Vol. 10, pp. 413-447). Stamford, CT: JAI Press.

Ryan, R. M., \& Deci, E. L. (2000). Intrinsic and extrinsic motivations: Classic definitions and new directions. Contemporary Educational Psychology, 25(1), 54-67. https://doi.org/10.1006/ ceps. 1999.1020

Ryan, R. M., \& Deci, E. L. (2017). Self-determination theory: Basic psychological needs in motivation, development, and wellness. Guilford Publications. https://doi.org/10.1521/978. $14625 / 28806$

Shellock, F. G., \& Prentice, W. E. (1985). Warming-up and stretching for improved physical performance and prevention of sports-related injuries. Sports Medicine, 2(4), 267-278. https://doi.org/10.2165/00007256-198502040-00004

Smith, C. A. (1994). The warm-up procedure: To stretch or not to stretch. A brief review. Journal of Orthopaedic \& Sports Physical Therapy, 19(1), 12-17. https://doi.org/10.2519/ jospt.1994.19.1.12

Spink, K. S., \& Roberts, G. C. (1980). Ambiguity of outcome and causal attributions. Journal of Sport Psychology, 2, 237-244. https://doi.org/10.1123/jsp.2.3.237

Stewart, D. W., \& Shamdasani, P. N. (1990). Focus groups: Theory and practice. Newbury Park, CA: Sage Publications.

Stewart, I. B., \& Sleivert, G. G. (1998). The effect of warm-up intensity on range of motion and anaerobic performance. Journal of Orthopaedic \& Sports Physical Therapy, 27(2), 154-161. https://doi.org/10.2519/jospt.1998.27.2.154

Swanson, J. R. (2006). A functional approach to warm-up and flexibility. Strength and Conditioning Journal, 28(5), 30-36. https://doi.org/10.1519/00126548-200610000-00005

Taylor, K. L., Sheppard, J. M., Lee, H., \& Plummer, N. (2009). Negative effect of static stretching restored when combined with a sport specific warm-up component. Journal of 


\section{Macrothink}

Science and Medicine in Sport, 12(6), 657-661. https://doi.org/10.1016/j.jsams.2008.04.004

Thompsen, A. G., Kackley, T. E. D., Palumbo, M. A., \& Faigenbaum, A. D. (2007). Acute effects of different warm-up protocols with and without a weighted vest on jumping performance in athletic women. Journal of Strength and Conditioning Research, 21(1), 52-56. https://doi.org/10.1519/00124278-200702000-00010

Woods, K., Bishop, P., \& Jones, E. (2007). Warm-up and stretching in the prevention of muscular injury. Sports Medicine, 37(12), 1089-1099. https://doi.org/10.2165/00007256-2007 37120-00006

Yıldırım, A., \& Şimşek, H. (2005). Sosyal bilimlerde nitel araştırma yöntemleri. Ankara: Seçkin Yayıncılık.

Zois, J., Bishop, D. J., Ball, K., \& Aughey, R. J. (2011). High-intensity warm-ups elicit superior performance to a current soccer warm-up routine. Journal of Science and Medicine in Sport, 14(6), 522-528. https://doi.org/10.1016/j.jsams.2011.03.012

\section{Copyright Disclaimer}

Copyright for this article is retained by the author(s), with first publication rights granted to the journal.

This is an open-access article distributed under the terms and conditions of the Creative Commons Attribution license (http://creativecommons.org/licenses/by/3.0/). 